\title{
A System for Relieving Mental Fatigue Based on Light and Sound Stimulation

\author{
Yang Zhang ${ }^{1, a}$, Xin Geng ${ }^{2, b}$, Hong Wang ${ }^{3, c}$ \\ 1,2,3 School of Mechanical Engineering and Automation, Northeastern University(110819), \\ Shenyang, Liaoning, China \\ a Legolas.Loire@gmail.com, ${ }^{\mathrm{b}}$ dbdx19871949@gmail.com, ${ }^{\mathrm{c}}$ hongwang@mail.neu.edu.cn
}

Keywords: Light and sound stimulation, Signal modulation, Spectral analyzing, Synchronizing

Abstract. A kind of stimulation device aiming at alleviating brain fag was designed. Meanwhile, the specific stereo signal which is expected to induce alpha-wave of human brain was programmed and encoded into audio files. To evaluating the effectiveness of this system, a comparison test was conducted. Volunteers are divided into control group and experimental group. Via power spectral analyzing, we can find the difference of EEG signals between control group and experimental group in alpha band $(8 \sim 13 \mathrm{~Hz})$. It can be noted that the intensity of alpha-wave and beta-wave of experimental group is larger than that of control group obviously.

\section{Introduction}

Human brain consists of tremendous amounts of neurons and glial cells. Two kinds of brain electrical activity can be recorded by electrophysiological method: evoked cortical potential and spontaneous electrical activity of brain. In clinic, the waveform diagram which is recorded on the scalp via electronic amplifier is called electroencephalogram (EEG). This diagram can reflect the spontaneous electrical activity of brain.

The EEG can be divided into four sections according to the frequency: Alpha-wave $(8-13 \mathrm{~Hz}$, $20-100 \mathrm{uV}$ ) is the main waveform when an adult is under resting condition. Beta-wave (14-30Hz, $5-20 \mathrm{uV}$ ) can be obvious when an adult open his eyes or is under stress, this waveform can be observed at frontal and parietal. Theta-wave $(4-7 \mathrm{~Hz}, 100-150 \mathrm{uV})$ is a mark of tiredness, when an adult is getting tired, theta-wave can be recorded at frontal and parietal clearly. Delta-wave $(0.5-3 \mathrm{~Hz}, 20-200 \mathrm{uV})$ can hardly be observed when an adult is in consciousness. It appears when a person is asleep or given anesthesia[1].

According to the information above, we can see that alpha-wave and beta-wave can reflect a person's mental state. Some former experimental results show that irritation of signals with rhythm on eyes and/or ears have impact on EEG signal in specific frequency [2]. This is the fundamental of L\&S Stimulation. Actually, there are many ways to exert simulation on sensory organs, such as optical, audio and somatosensory[3]. But the most accepted method is audio stimulation. Currently, the main research topic in this field is in what way can stimulation have the most noticeable but safe effect on human brain. These ways differ in the frequency, amplitude and media of the stimulation. In this paper, we will design a stimulating system and do some experiments based on these questions.

\section{Hardware Design}

To accomplish this experiment, we need a stimulating system. This system must be able to generate audio signals and synchronize optical signals with sound since we adopt the method of light and sound stimulation. So the hardware system can be divided into two modules: audio signal generating circuit and light synchronizing circuit. The following part of this section will have an elaborate description of the system. 
Audio Signal Generating Circuit. Considering about the variety and complexity of the audio signal, an audio codec should be a good choice to re-product the signal. In this system, we choose SC95869 (Silan Microelectronics CO., LTD) as the kernel of audio codec circuit. SC95869 is a specific audio codec chip using 51 series MCU as its processing unit and has many internal resources such as ADC (for audio input), DAC (for audio output), OTP (One Time Programmable) chip or ROM (to store executable code). Meanwhile, it provides abundant peripheral interfaces to perform a MP3 Player's function, such as IIS, key/LCD, mass storage (USB, SD, Nandfalsh), etc[4]. Fig. 1 shows the architecture of SC95869.

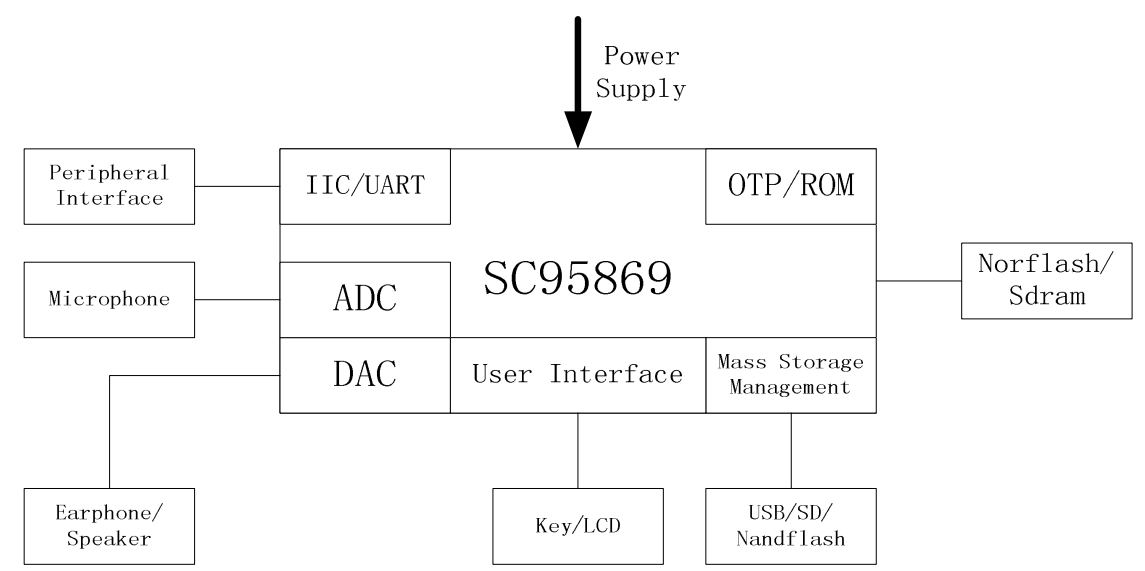

Fig. 1. Architecture of SC95869

The codec circuit should fulfill the following function: It has firmware that can decode audio files (wav and mp3 format) stored in ROM. When getting audio files, it can decode and transform them into audio signal, finally send to earphones. Users can control it by pushing some buttons.

Allow for all the functions above, we need DAC, MSM (Mass Storage Management) interfaces, the other ports is used as GPIO (General Purpose Input and Output) ports which is connected to keypad and LCD display. The MSM module supports multiple storage media, such as USB device, SD card and Nan flash. But USB device is more convenient in data transmitting with computers than the other two kinds of media. So we use USB devices as our storage media to store audio files. Since SC95869 has ROM in it and the storage capacity is adequate, we don't need extend the storage space.

Light Synchronizing Circuit. The key point for L\&S Stimulation is synchronizing the strength and frequency of light with sound. The changing of sound is reflected by the changing of output voltage via DAC. So we proposed a method that optical signal is modulated by audio signal directly. An ASIC (Application Specific Integrated Circuit) called KA2284 (SAMSUNG Electronics CO,. LTD) is adopted in this circuit. The KA2284 is monolithic integrated circuit designed for 5-dot LED level meters with a built-in rectifying amplifier. It's suitable for AC/DC level meters such as VU meters or signal meters. Fig .2 shows the block diagram and typical application circuit[5].

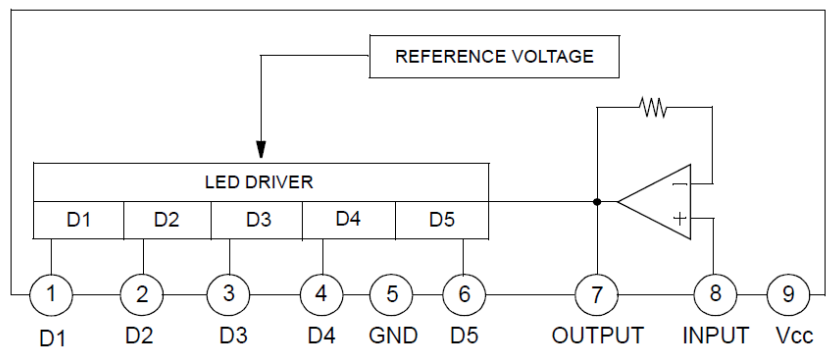

Fig. 2(a) Block Diagram of KA2284 


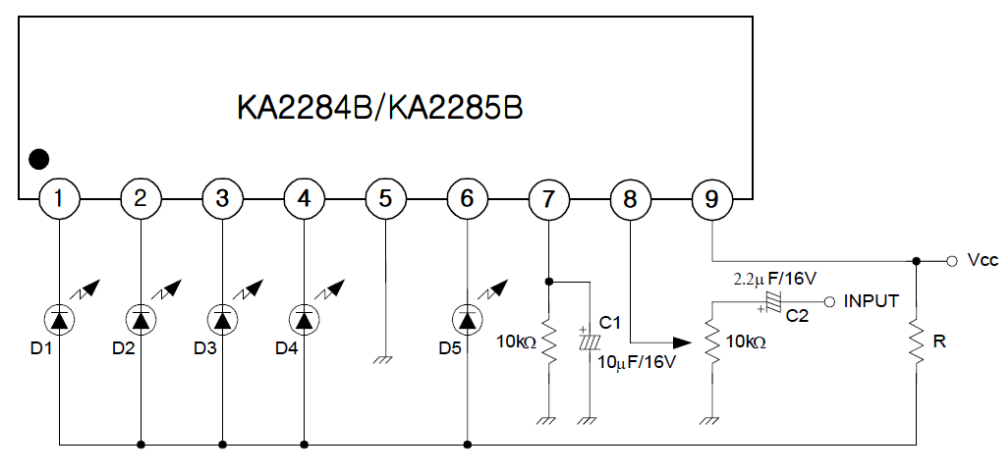

Fig. 2(b) Typical Application Circuit

KA2284 is a SIP chip with nine pins. Pin 1, 2, 3, 4 and 6 are connected to LEDs, pin 8 is connected to input signal (When connected to DC signals, the capacitor C2 is not necessary). When powered up, KA2284 will light different number of LEDs according to the amplitude of input signal. The lighten sequence of LEDs is D1, D2, D3, D4, D5, i.e. the threshold voltage which is needed to light D2 is higher than that of D1, etc. In this application, we use three LEDs connected to pin 2, 3 and 4 to get obvious flickers.

Fig. 3 shows the block diagram of the system.

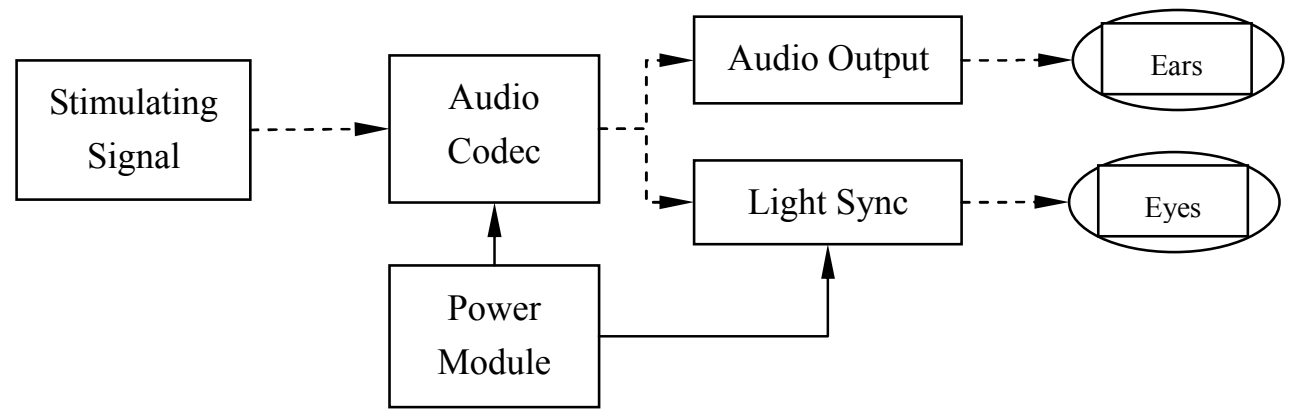

Fig. 3. Block Diagram of the System

\section{Software Design}

This section contains two parts: development of audio player and development of audio files. The audio player program is based on the hardware system mentioned above, accomplishing the simple function of audio player. The audio files are programmed using MATLAB.

Audio Player. To getting better performance and shortening developing period, Silan Microelectronics provides BSP (Board Support Package) with a wealth of API (Application Programming Interfaces) based on SC9586X series. Fig. 4 lists all the libraries provided and the dependencies. The developing tool is Keil. Users just need include the library files in the project to get access to the functions provided[6]. 


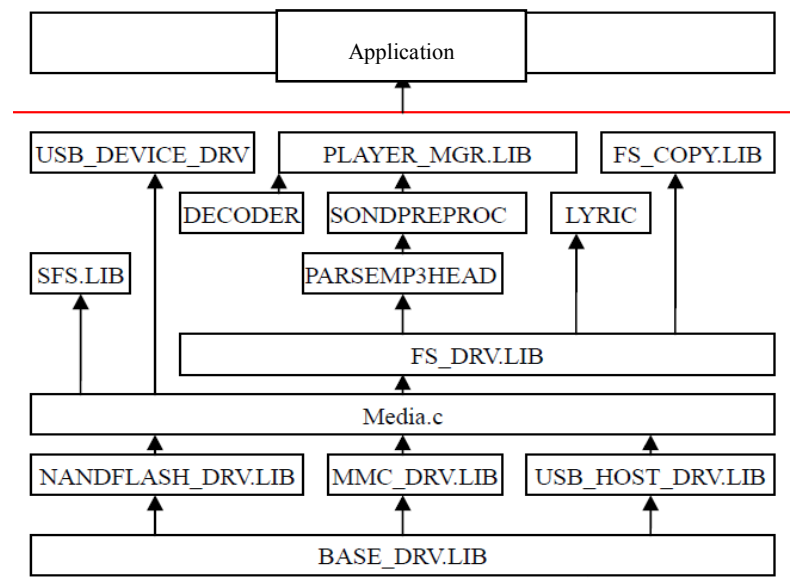

Fig. 4. Libraries and Dependencies

Modulation and Design of Audio Files. Three kinds of audio files are designed:

a): Pure alpha-band wave with frequency following Gaussian Distribution. Alpha-wave ranges between 8 to $13 \mathrm{~Hz}$, which cannot be heard by human ears. So we adopt the method of modulating[7]. The following lists MATLAB program and the waveform and spectrum of the audio signal is shown in Fig. 5:

clear all; close all;

$\mathrm{fs}=44100 ; \mathrm{n}=\left[1: 2^{\wedge} 16\right]$;

wav $=1+\sin \left(n * 2 * \mathrm{pi}^{*}(8+5 *\right.$ rand $\left.) / \mathrm{fs}\right)$

base $=\sin \left(\mathrm{n} * 2 * \mathrm{pi}^{*} 220 / \mathrm{fs}\right)+0.5 * \sin (\mathrm{n} * 2 * \mathrm{pi} * 200 / \mathrm{fs})+0.5 * \sin (\mathrm{n} * 2 * \mathrm{pi} * 250 / \mathrm{fs})$;

output=wav.*base;

soundsc(output,fs);

fout $=\mathrm{fft}($ output,fs);

mout $=$ abs(fout);

frq=linspace $(0, \mathrm{fs}, \mathrm{fs})$;

subplot(2,1,1); plot(frq,output(1:fs));

title('Waveform of Modulating Signal'), xlabel('Samples');

$\operatorname{subplot}(2,1,2) ; \operatorname{plot}(\operatorname{frq}(1: 300), \operatorname{mout}(1: 300))$;

title('Spectrum of Modulating Signal'), xlabel('Frequency/Hz');

wavwrite( $0.25 *$ output,fs,'Alpha_Modulate');
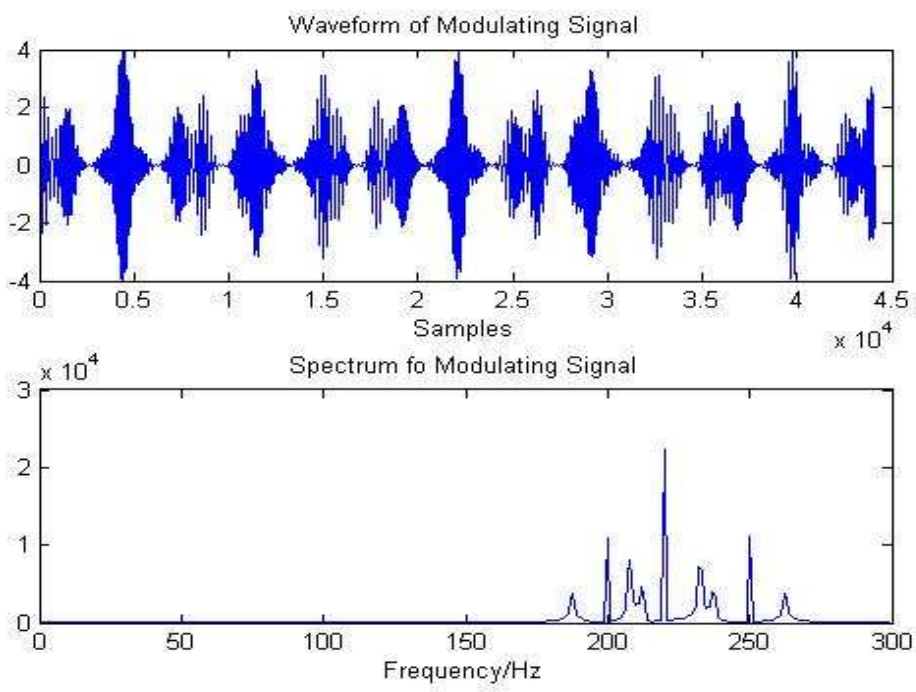

Fig. 5. Waveform and Spectrum of the Audio Signal 
b): Alpha-band wave with background music. The theory is similar to a), but it makes the sound less rigid.

c): Signals with frequency difference between left and right ear. That means the signals sent to left and right ears are different, but the difference of these signals are in alpha-band. Some existing results show that this kind of stimulation may lead to a state of "Brain-Sync"[8], finally promote working efficiency.

\section{Experiment and Results}

To evaluating the effectiveness of this system, a comparison test was conducted. Volunteers are divided into control group and experimental group. Before the test begins, researchers record the EEG (Electroencephalogram) signals of both groups. Then each group's volunteers drive on the driving simulator for two hours to achieve a state of mental fatigue, and record the EEG signals. After driving, the volunteers in experimental group wear this device for $20 \mathrm{mins}$, while the counterpart in control group just sit and relax for 20mins. Finally record the EEG signals for both groups.

The electrodes were placed according to the 10-20 international system as depicted in Fig. 6.

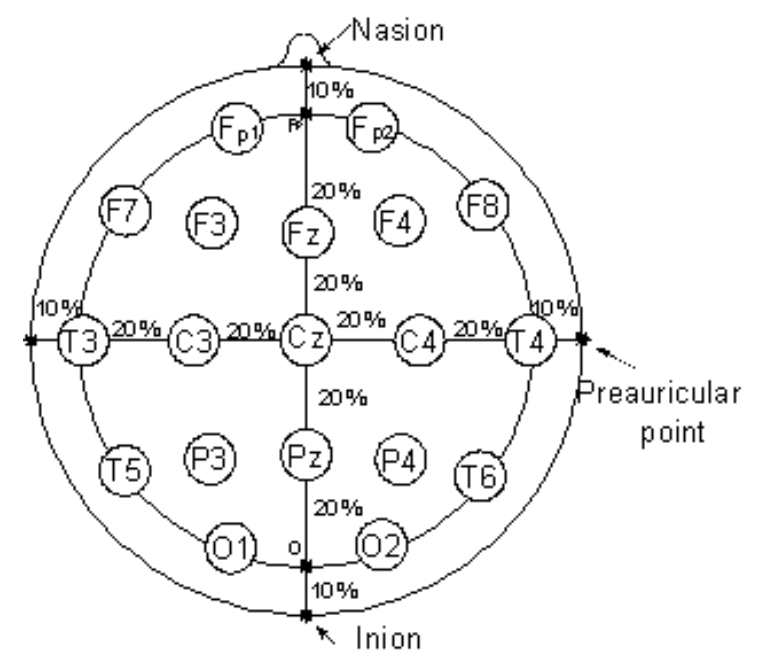

Fig. 6. The 10-20 System of Electrode Placement

The data for this experiment were taken using the neuroscience facilities at Northeastern University, which are located in a laboratory. The subject was seated in a comfortable chair. A computer running the program used to present visual stimulation to the subject, NeuroScan Inc.'s SCAN 4.0. A QuikCap-40 amplifier was used to record EEG signals from positions C3, C4, P3, P4, O1, and O2, shown in Fig. 6 and defined by the 10-20 system of electrode placement. These 6 channels were referenced to electrically linked mastoids at A1 and A2. The impedance of all electrodes was kept below $5 \mathrm{~K}$ ohms. The data were recorded at a sampling rate of $1000 \mathrm{~Hz}$ with a SynAmps2 Model EEG amplifier, which uses a 32 bit A/D converter. A serial cable connected the computer used to present the stimulation to the SynAmps2 EEG amplifier, and was used to signal when a stimulus was presented. The SynAmps 2 was programmed to do analog band-pass filtering from $0.5-100 \mathrm{~Hz}$, and was calibrated with a known voltage before the recording session[9].

Fig. 7(a) shows the EEG signal acquired. The quality of the EEG data depends on several conditions, such as electrode positioning and the subjects' behavior. In this study, artifacts and noises were removed from the signal sets by filtering and visual inspection. 


\section{Conclusion}

Fig. 7(b) shows the relation between frequency and power spectral density of the EEG signal acquired. It can be noted that the intensity of alpha-wave and beta-wave of experimental group is larger than that of control group obviously. The intensity of alpha-wave and beta-wave of the experimental group is nearly equal, while the intensity of alpha-wave of the control group is large than that of beta-wave[10].

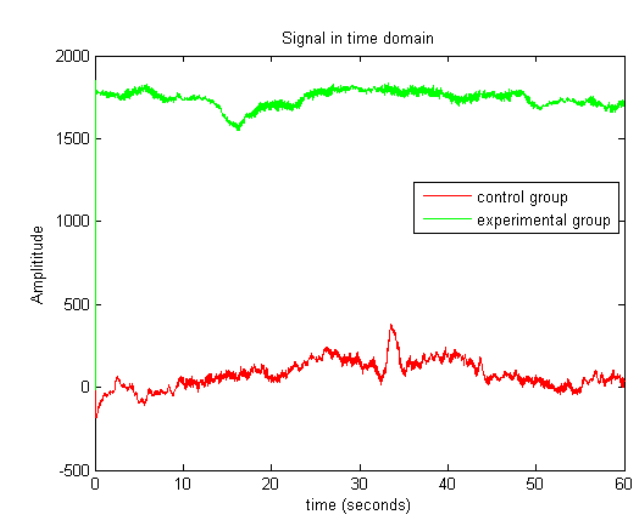

(a)

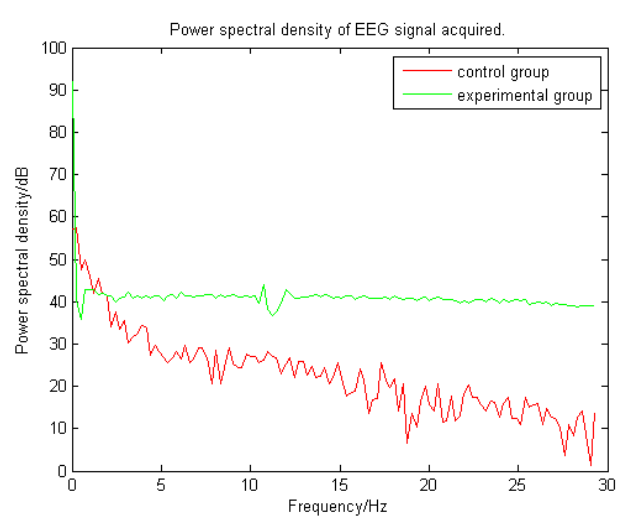

(b)

Fig. 7. Relation between frequency and power spectral density of the EEG signals

\section{References}

[1] Sun Qingwei, Zhou Guangji, Bai Jie, Human Physiology, China Medical Science Press, Beijing, 2009, pp. 356-360.

[2] Wu Min, Light and Brain-Sync Treatment, in: Adjustment and Treatment for Depression, Shanghai Science and Technology Press, Shanghai, 2009, pp. 117-118.

[3] Wang Lei, Wang Jiang, Liu Lifang, Yang Shuo, Xu Guizhi, Yang Weili, On-line Brain-computer Interface System Design Based on Alpha wave and Motor Imagery, Journal of Clinical Rehabilitative Tissue Engineering Research, 15(2011) 3152-3155.

[4] SC9586X Series Chip Datasheet, Silan Microelectronics CO., LTD, 2008.

[5] KA2284 Datasheet, SAMSUNG Electronics CO,. LTD.

[6] SC9586X Series Developing Handbook, Silan Microelectronics CO., LTD, 2008.

[7] Zhang Shuiying, Xu Weiqiang, Ji Li, Communication Fundamental, XDU Press, Xi'an, 2008, pp. $39-42$.

[8] Information on http://www.success001.com/menluo.htm.

[9] NeuroScan Product Release Note, Compumedics NeuroScan Inc.

[10] Wang Yongxuan, Qiu Tianshuang, Liu Rong, A Wavelet Analysis Method for Single Channel Evoked Potential Extraction with a Few Sweeps, Chinese Journal of Biomedical Engineering, 15(2011) 34-39. 